\title{
Left atrial appendage closure in patients with intracranial hemorrhage
}

\author{
Jalaj Garg ${ }^{1}$ (1) Siddharth Shah ${ }^{2} \cdot$ Kuldeep Shah $^{3} \cdot$ Rahul Bhardwaj $^{1} \cdot$ Tahmeed Contractor $^{1} \cdot$ Ravi Mandapati $^{1}$. \\ Mohit K. Turagam ${ }^{4} \cdot$ Andrea Natale $^{5} \cdot$ Dhanunjaya Lakkireddy $^{6}$
}

Received: 19 December 2021 / Accepted: 27 January 2022 / Published online: 2 February 2022

(c) The Author(s), under exclusive licence to Springer Science+Business Media, LLC, part of Springer Nature 2022

\section{Introduction}

Oral anticoagulation (OAC) has been the mainstay of therapy to reduce the risk of systemic thromboembolism in patients with atrial fibrillation (AF) [1]. More recently, percutaneous left atrial appendage closure (LAAC) has become an important treatment option in patients with AF to reduce their risk of thromboembolism, especially in those with a high risk of bleeding on long-term anticoagulation [2, 3]. However, most LAAC devices require patients to tolerate short-term anticoagulation or dual antiplatelet therapy (DAPT). For the same reason, the two landmark trials (PROTECT and PREVAIL trials), which resulted in the approval of Watchman (Boston Scientific, St Paul, MN) LAAC device by the Food and Drug Administration (FDA), had excluded patients with a prior history of intracranial hemorrhage (ICH) [2, 3].

Patients with prior ICH and AF have an increased risk of recurrent bleeding (which may prove life-threatening) and hence are often not treated with OAC and left unprotected. Nonetheless, they often also have a high risk of ischemic stroke. While the need for a short duration of OAC and/ or DAPT following LAAC remains a challenging situation,

Jalaj Garg

garg.jalaj@yahoo.com

1 Division of Cardiology, Cardiac Arrhythmia Service, Loma Linda University Health, 11234 Anderson St, Loma Linda, CA 92354, USA

2 Division of Cardiology, Cardiac Arrhythmia Service, Indiana University School of Medicine, Indianapolis, IN, USA

3 Department of Cardiovascular Medicine, Beaumont Hospital, Oakland University William Beaumont School of Medicine, Royal Oak, MI, USA

4 Helmsley Electrophysiology Center, Icahn School of Medicine at Mount Sinai, New York, NY, USA

5 Texas Cardiac Arrhythmia Institute at St. David's Medical Center, Austin, TX, USA

6 Kansas City Heart Rhythm Institute and Research Foundation, Kansas City, KS, USA
LAAC may still be an attractive solution for protection against thromboembolism in these patients. Recent studies have shown promising data regarding the use of percutaneous LAAC devices. We aimed to perform a pooled analysis to assess the safety and efficacy of LAAC in AF patients with prior ICH.

\section{Methods}

\subsection{Search strategy, study selection, and data extraction}

Electronic databases were searched from inception until June 15,2021 , using the keywords "left atrial appendage closure/ occlusion" and "Intracranial bleed." This systematic review was performed according to the PRISMA guidelines, and the study was prospectively enrolled in PROSPERO (ID 276,020).

The eligibility criteria for our systematic review and meta-analysis included (1) all studies reporting outcomes of LAAO in patients with ICH and (2) studies that included human subjects. We included studies only in the English language. Case reports, abstracts, editorial, or systematic reviews were excluded. Two investigators (JG and SS) independently performed the literature search and screened all titles and full-text versions of all relevant studies that met study inclusion criteria. The data from the included studies were extracted using a standardized protocol and a data extraction form. Any discrepancies between the two investigators were resolved with a consultation with the senior investigator (DL).

\subsection{Statistical analysis}

We used Freeman Tukey double arcsine method to establish variance of raw proportions. DerSimonian-Laird randomeffect model was used to combine the transformed proportions. Finally, we then back-transformed the pooled estimates 
and plotted the data on the forest plot. Heterogeneity of the effect size among the included studies was assessed by Higgins I-squared $\left(\mathrm{I}^{2}\right)$ statistic. A value of $\mathrm{I}^{2}$ of $0-25 \%$ represented insignificant heterogeneity, $26-50 \%$ represented low heterogeneity, $51-75 \%$ represented moderate heterogeneity, and more than $75 \%$ represented high heterogeneity, as set forth by the Cochrane Collaboration. A two-tailed $p<0.05$ was considered statistically significant for all analyses. The entire meta-analysis was performed using a meta-package for $\mathrm{R}$ version 4.0 and RStudio version 1.2.

\subsection{Outcomes}

The outcomes studied were (1) acute procedure success, (2) periprocedural complications (within 7 days), (3) post-procedure complication ( $>7$ days), and (4) all-cause mortality.

\section{Results}

A total of 44 citations were identified during the initial search. After a detailed evaluation, 37 records were excluded, and 7 studies were included in the final analysis (Fig. 1). Seven retrospective studies including $407 \mathrm{ICH}$ patients who underwent LAAC met study inclusion criteria [1, 4-9]. The mean follow-up was $14.6 \pm 10.1$ months, mean age was $74.2 \pm 6.8$ years, and $35.6 \%$ were females. Mean $\mathrm{CHA}_{2} \mathrm{DS}_{2} \mathrm{VASC}$ and HAS-BLED scores were $4.8 \pm 1.5$ and $4 \pm 1$, respectively. A total of $66.3 \%(n=270)$ patients underwent LAAC with Amplatzer Cardiac plug, 32.4\% $(n=132)$ received Watchman, and $1.2 \%(n=5)$ patients received Amulet device. Time to LAAC from ICH was $452.3 \pm 652.3$ days (Table 1 ). Acute procedure success was achieved in $98.5 \%$ patients. Periprocedural complications observed were pericardial effusion $0.17 \%$ (95\% CI 0-0.87), device embolization $0.1 \%$ (95\% CI 0-0.95), device-related thrombosis (DRT) $0.03 \%$ (95\% CI 0-1.44), major bleeding $0.02 \%$ (95\% CI 0-0.83), and recurrent ICH 0\% (95\% CI 0-0.56\%) (Fig. 2A). Post-procedure complications observed during the follow-up period included major bleeding $0.25 \%$ (95\% CI 0-1.75), recurrent ICH $0.05 \%$ (95\% CI 0-0.96), ischemic stroke $0.54 \%$ (95\% CI 0-3.31), DRT 0.49\% (95\% CI $0-1.54)$, and all-cause mortality $1.4 \%$ (95\% CI 0-4.72). There was no device embolization during the follow-up period (Fig. 2B).

\section{Discussion}

To the best of our knowledge, this is the first systematic review evaluating the outcomes of LAAC in AF patients with prior ICH and AF. The LAAC was successfully achieved in $98.5 \%$ of patients with a low risk of periprocedural and post-procedure complications-findings comparable to prior landmark trials with LAAC in the general population. High-dose intravenous heparin bolus administered
Fig. 1 Flow diagram illustrating the systematic search of studies

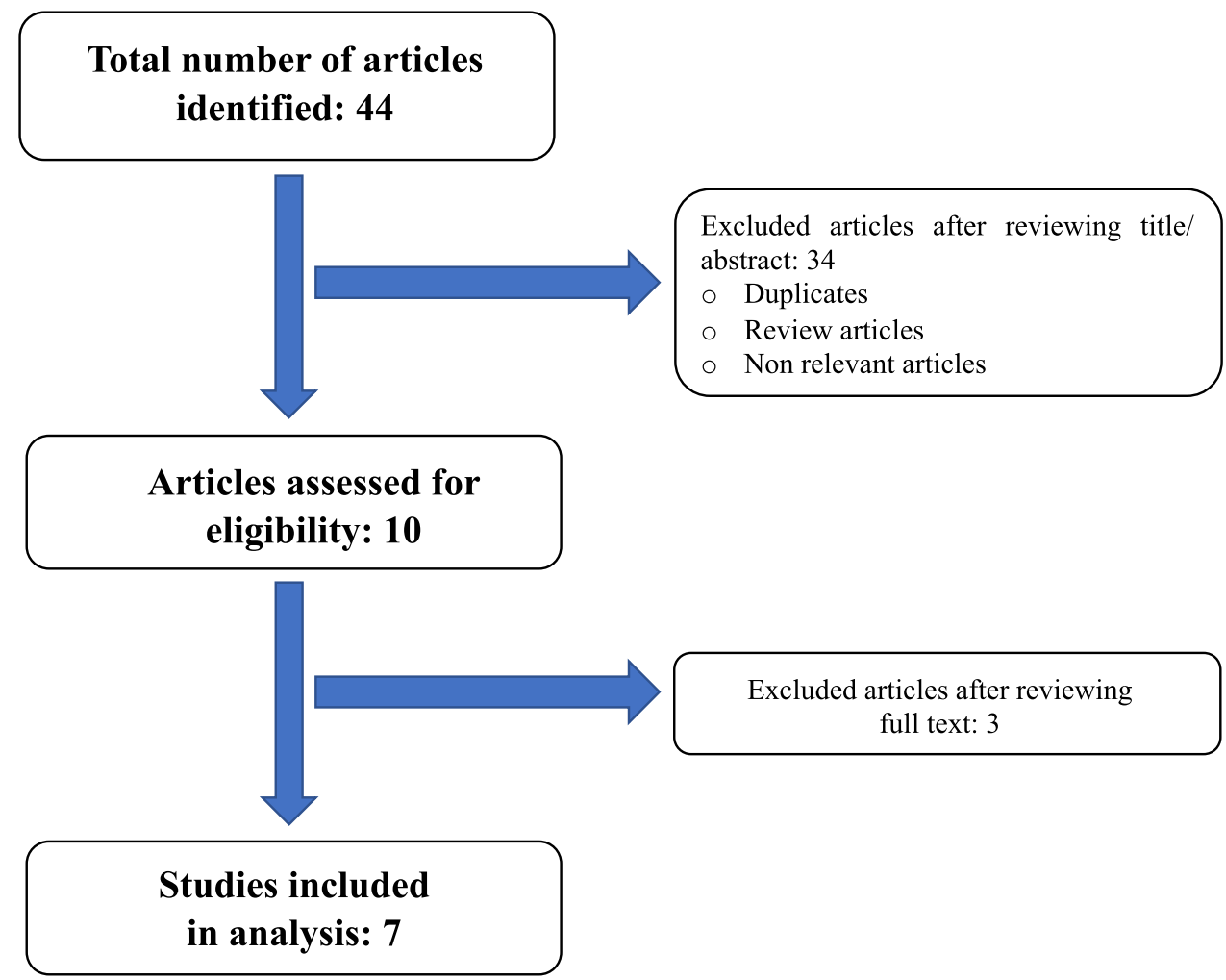




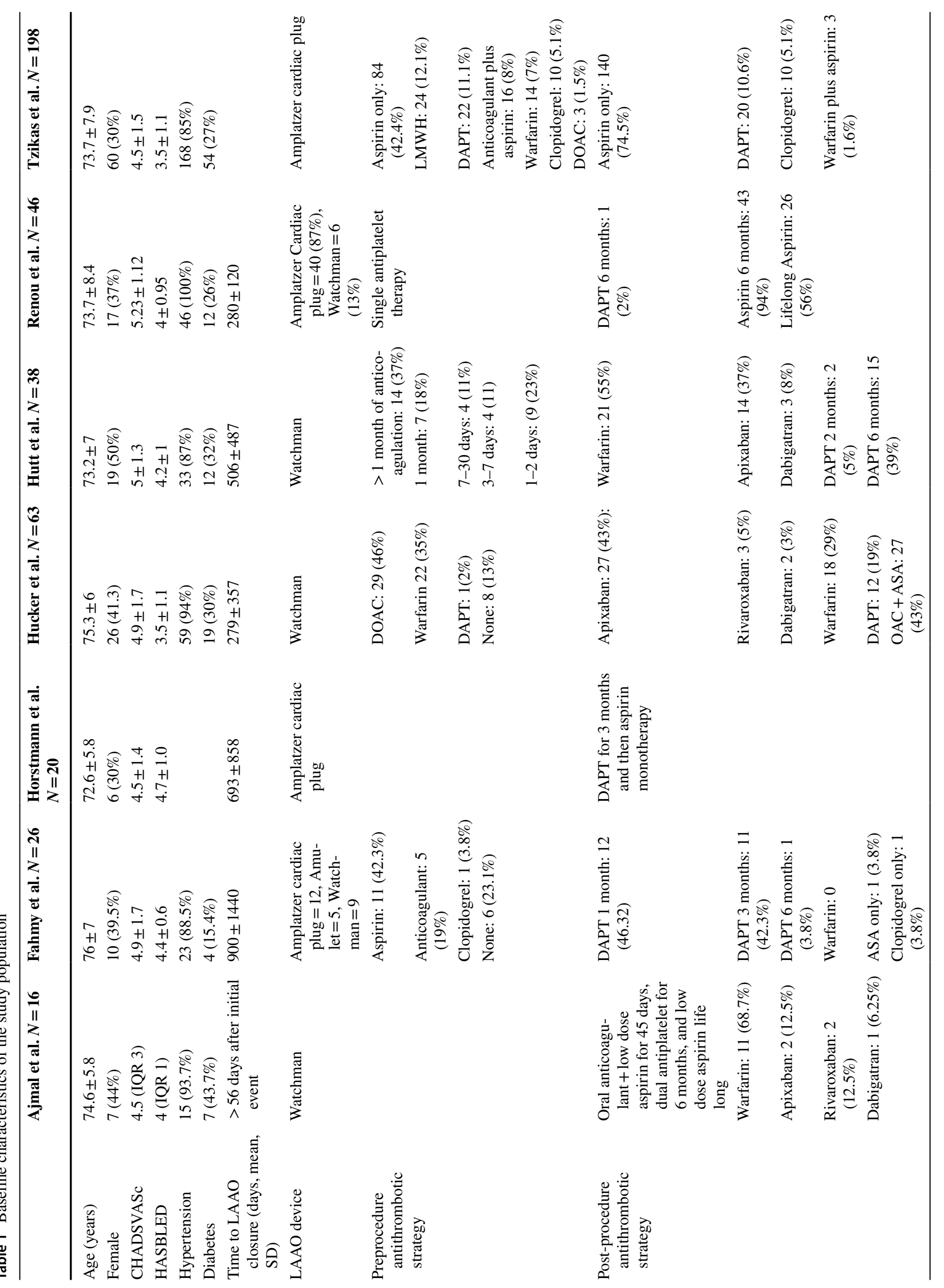




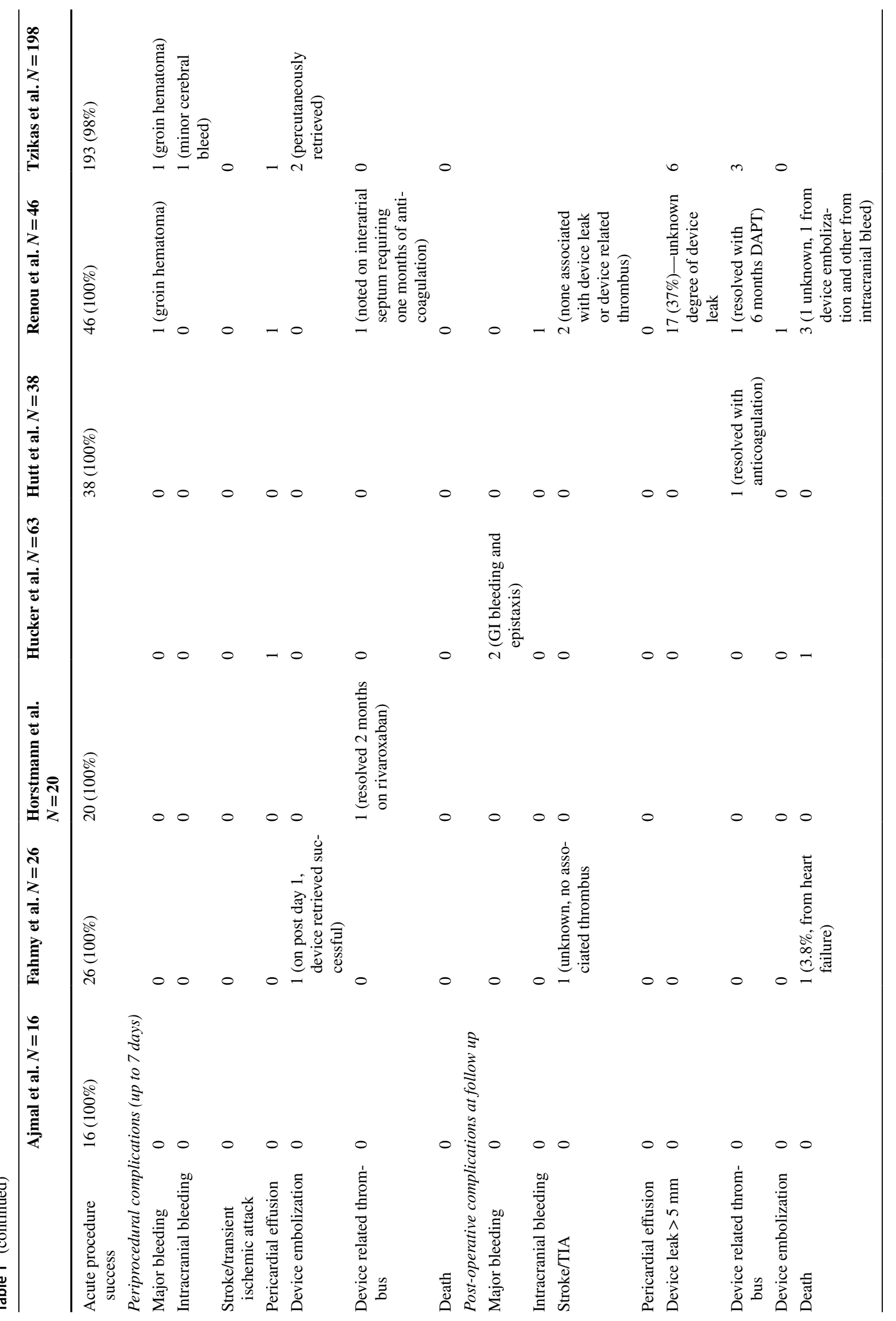




\section{Summary plot of clinical outcomes}
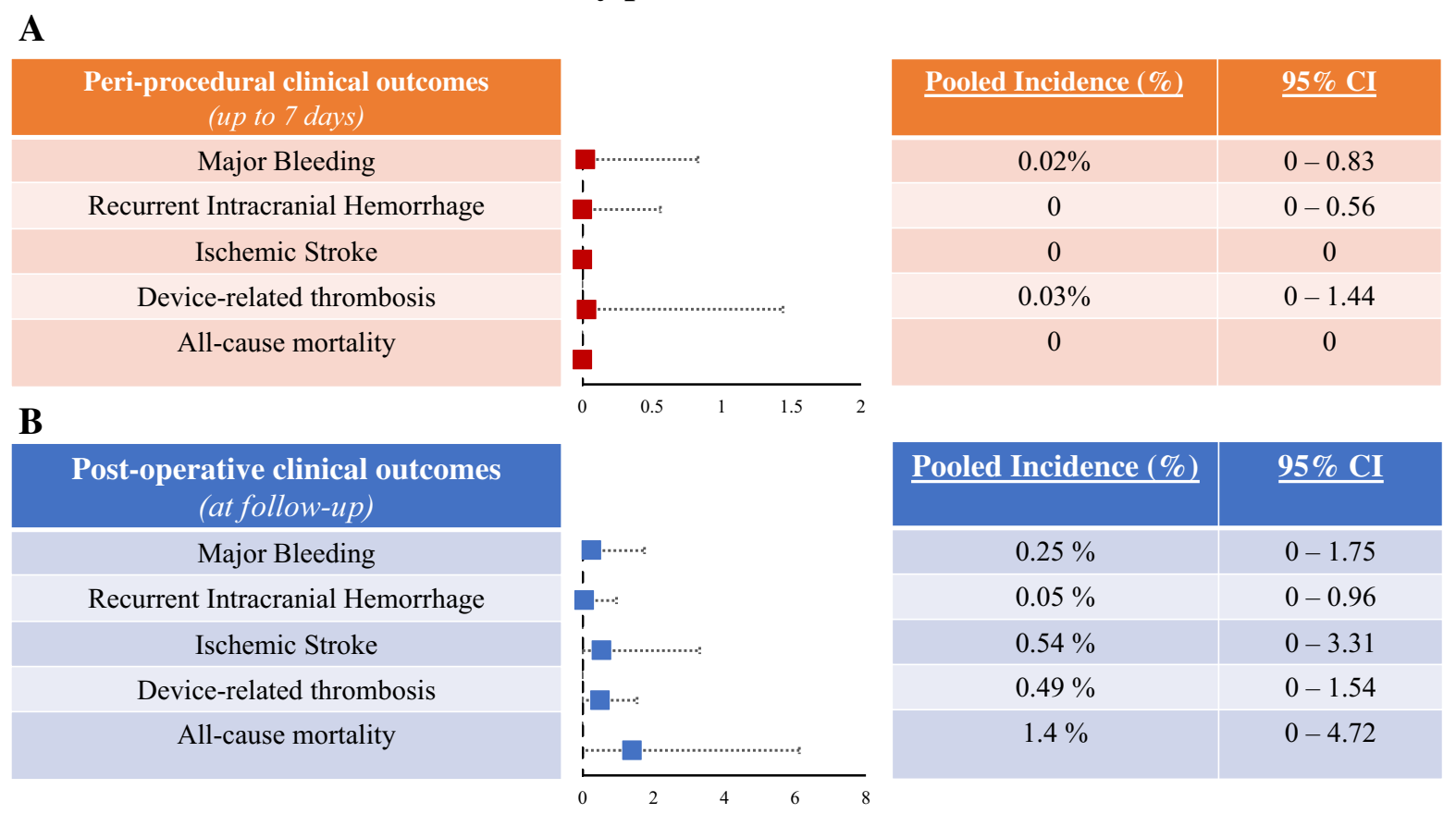

\begin{tabular}{|c|c|}
\hline Pooled Incidence (\%) & 95\% CI \\
\hline $0.25 \%$ & $0-1.75$ \\
\hline $0.05 \%$ & $0-0.96$ \\
\hline $0.54 \%$ & $0-3.31$ \\
\hline $0.49 \%$ & $0-1.54$ \\
\hline $1.4 \%$ & $0-4.72$ \\
\hline
\end{tabular}

Fig. 2 Summary plot of clinical outcomes. Panel A: Periprocedural clinical outcomes (up to 7 days). Panel B: Postoperative clinical outcomes (at follow-up)

during the LAAC procedure increases the risk of periprocedural major bleeding or ICH in this high-risk cohort. However, the pooled incidence of periprocedural major bleeding and recurrent ICH was low in our study $(0.02 \%$ and $0 \%$, respectively). The anti-thrombotic regimen following LAAC (typically with Watchman device) (until the endotheliazation is completed) is variable across countries (OAC is preferred in the USA for initial 6 weeks vs. DAPT in Europe for initial $1-3$ months). Only $26.3 \%$ of patients in our study received OAC post-LAAC, while the rest received single or dual antiplatelet therapy. The pooled incidence of DRT at follow-up was $0.49 \%$ (lower than previous studies) - likely driven by the fact that two-thirds of patients underwent LAAC via the device type that does not require OAC post-procedure. In addition, of patients who underwent LAAC via Watchman device, only $78.8 \%$ of patients received post-implantation OAC (104 of 132). Patients with prior ICH hypothetically would have difficulty with an additional short course of OAC should a DRT be detected. Of 6 patients total with DRT (LAAC type - 4 Amplatzer Cardiac plug, 1 Watchman, 1 unknown), one patient had DRT within the first 7 days postLAAC (received Amplatzer Cardiac plug) (successfully resolved with 2 months rivaroxaban with no recurrent $\mathrm{ICH}$ ), and 5 had DRT during the follow-up period - two of which (one with Watchman and another device type unknown, respectively) DRT was successfully resolved with OAC and DAPT, respectively. The treatment strategy of the other three
DRT patients (all received Amplatzer Cardiac plug) was unavailable. None of the patients with DRT experienced stroke/TIA, either periprocedural or post-procedure.

In addition, there exists no consensus on restarting OAC (either DOAC or warfarin) in AF patients with ICH, and therefore, LAAC appears to be a suitable alternative. In our study, the pooled incidence of major bleeding and recurrent ICH at long-term follow-up was $0.25 \%$ and $0.05 \%$, respectively (much lower than the anticipated bleeding risk with OACs for the mean HAS-BLED score of 4 in our study). Similarly, the pooled incidence of ischemic stroke at $0.54 \%$ at follow-up (significantly lower than the anticipated stroke risk without OAC for a mean $\mathrm{CHA}_{2} \mathrm{DS}_{2}$ VASC of 4.8). Taken together, our study provides the best available evidence to date and suggests that LAAC is a safe and effective therapeutic option in patients with AF and prior history of ICH with an acceptable periprocedural and post-procedure risk (although the findings of the study could be primarily driven by the fact that time to LAAC from index ICH was 452 days-further mitigating periprocedural and postprocedural complications).

Future randomized controlled trial (RCT) with enough sample size might shed light on the true clinical benefit. Relevant RCT-STROKECLOSE (clinicaltrial.gov NCT02830152 - 2:1 randomization strategy against medical therapy) is currently ongoing with the primary endpoint composite of stroke (ischemic or hemorrhagic), systemic 
embolism, life-threatening or major bleeding, and allcause mortality, with a time frame up to 5 years after randomization in AF patients with prior ICH. In addition, the most optimal anti-thrombotic therapy post-LAAC in ICH patients remains to be determined. An ongoing trial evaluating the outcomes on single antiplatelet therapy following LAAC with Watchman is underway and may provide further insight, which may especially prove beneficial in this high-risk population [10]. While several patients in our pooled analysis received aspirin monotherapy post-LAAC, individual patient-level data were not available to evaluate the differences in bleeding events with different antithrombotic/antiplatelets. This meta-analysis is limited by operatorpatient selection bias, small sample size, lack of patient-level data, retrospective study design, heterogeneous follow-up length, time to LAAC after ICH, and lack of brain imaging to assess the etiology of ICH and its stability. Another area lacking the data is regarding the optimal antithrombotic/ antiplatelet regimen post-LAAC in AF patients with ICH patients and warrants further investigation.

\section{Conclusion}

LAAO is a safe and effective therapeutic option in patients with AF and prior history of ICH with an acceptable periprocedural and post-procedure risk.

\section{Declarations}

Competing interests The authors declare no competing interests.

\section{References}

1. Fahmy P, Spencer R, Tsang M, Gooderham P, Saw J. Left Atrial appendage closure for atrial fibrillation is safe and effective after intracranial or intraocular hemorrhage. Can J Cardiol. 2016;32:349-54.
2. Holmes DR Jr, Kar S, Price MJ, Whisenant B, Sievert H, Doshi SK, Huber K, Reddy VY. Prospective randomized evaluation of the Watchman Left Atrial Appendage Closure device in patients with atrial fibrillation versus long-term warfarin therapy: the PREVAIL trial. J Am Coll Cardiol. 2014;64:1-12.

3. Holmes DR, Reddy VY, Turi ZG, Doshi SK, Sievert H, Buchbinder M, Mullin CM, Sick P, Investigators PA. Percutaneous closure of the left atrial appendage versus warfarin therapy for prevention of stroke in patients with atrial fibrillation: a randomised non-inferiority trial. Lancet. 2009;374:534-42.

4. Horstmann S, Zugck C, Krumsdorf U, Rizos T, Rauch G, Geis $\mathrm{N}$, Hardt S, Veltkamp R. Left atrial appendage occlusion in atrial fibrillation after intracranial hemorrhage. Neurology. 2014;82:135-8.

5. Ajmal M, Naik H, Kocheril A. Left atrial appendage closure in patients with intracranial hemorrhage and nonvalvular atrial fibrillation. J Stroke Cerebrovasc Dis. 2020;29:104685.

6. Hucker WJ, Cohen JA, Gurol ME, Heist EK, Gianni C, Galvin J, Atkins D, Bommana S, Di Biase L, Ruskin J, Mohanty S, Horton R, Lakkireddy D, Natale A, Mansour M. WATCHMAN implantation in patients with a history of atrial fibrillation and intracranial hemorrhage. J Interv Card Electrophysiol. 2020;59:415-21.

7. Hutt E, Wazni OM, Saliba WI, Kanj M, Tarakji KG, Aguilera J, Barakat AF, Rasmussen P, Uchino K, Russman A, Hussain S, Wisco D, Kapadia S, Lindsay BD, Hussein AA. Left atrial appendage closure device implantation in patients with prior intracranial hemorrhage. Heart Rhythm. 2019;16:663-8.

8. Renou P, Thambo JB, Iriart X, Nicot S, Kabore N, Jalal Z, Olindo S, Debruxelles S, Poli M, Rouanet F, Sibon I. Left atrial appendage closure in patients with atrial fibrillation and previous intracerebral hemorrhage. J Stroke Cerebrovasc Dis. 2017;26:545-51.

9. Tzikas A, Freixa X, Llull L, Gafoor S, Shakir S, Omran H, Giannakoulas G, Berti S, Santoro G, Kefer J, Aminian A, Gloekler S, Landmesser U, Nielsen-Kudsk JE, Cruz-Gonzalez I, Kanagaratnam P, Nietlispach F, Ibrahim R, Sievert H, Schillinger W, Park JW, Meier B, Karvounis H. Patients with intracranial bleeding and atrial fibrillation treated with left atrial appendage occlusion: results from the Amplatzer Cardiac Plug registry. Int J Cardiol. 2017;236:232-6.

10. Holmes DR, Reddy VY, Buchbinder M, Stein K, Elletson M, Bergmann MW, Schmidt B, Saw J. The assessment of the Watchman device in patients unsuitable for oral anticoagulation (ASAPTOO) trial. Am Heart J. 2017;189:68-74.

Publisher's note Springer Nature remains neutral with regard to jurisdictional claims in published maps and institutional affiliations. 